\title{
Dihydrobenzoic Acid Modified Nanoparticle as a MALDI-TOF MS Matrix for Soft Ionization and Structure Determination of Small Molecules with Diverse Structures
}

\author{
Mei-Chun Tseng, ${ }^{a}$ Rofeamor Obena, ${ }^{\mathrm{b}}$ Ying-Wei Lu, ${ }^{\mathrm{c}}$ Po-Chiao Lin, ${ }^{\mathrm{c}, \mathrm{d}}$ \\ Ping-Yu Lin, ${ }^{a}$ Yung-Sheng Yen, ${ }^{a}$ Jiann-Tsuen Lin, ${ }^{a}$ Li-De Huang, ${ }^{c}$ \\ Kuang-Lieh Lu, ${ }^{a}$ Long-Li Lai, ${ }^{\mathrm{f}}$ Chun-Cheng Lin, ${ }^{\mathrm{c}, \mathrm{d}}$ and Yu-Ju Chen ${ }^{\mathrm{a}, \mathrm{d}, \mathrm{e}}$ \\ ${ }^{a}$ Institute of Chemistry, Academia Sinica, Taipei, Taiwan \\ ${ }^{\mathrm{b}}$ Institute of Chemistry, University of the Philippines Diliman, Quezon, Philippines \\ ${ }^{c}$ Department of Chemistry, Tsing Hua University, Hsinchu, Taiwan \\ ${ }^{d}$ Chemical Biology and Molecular Biophysics, Taiwan International Graduate Program, Taipei, Taiwan \\ ${ }^{\mathrm{e}}$ Department of Chemistry, National Taiwan University, Taipei, Taiwan \\ ${ }^{\mathrm{f}}$ Department of Applied Chemistry, National Chi Nan University, Nantou, Taiwan
}

\begin{abstract}
Efficient structural characterization is important for quality control when developing novel materials. In this study, we demonstrated the soft ionization capability of the hybrid of immobilized silica and 2,5-dihydrobenzoic acid (DHB) on iron oxide magnetic nanoparticles in MALDI-TOF MS with a clean background. The ratio between $\mathrm{SiO}_{2}$ and $\mathrm{DHB}$ was examined and was found to affect the surface immobilization of DHB on the nanoparticle, critically controlling the ionization efficiency and interference background. Compared with commercial DHB, the functionalized nanoparticle-assisted MALDI-TOF MS provided superior soft ionization with production of strong molecular ions within $5 \mathrm{ppm}$ mass accuracy on a variety of new types of synthetic materials used for solar cells, light emitting devices, dendrimers, and glycolipids, including analytes with either thermally labile structures or poor protonation tendencies. In addition, the enhancements of the molecular ion signal also provided highquality product-ion spectra allowing structural characterization and unambiguous small molecule identification. Using this technique, the structural differences among the isomers were distinguished through their characteristic fragment ions and comprehensive fragmentation patterns. With the advantages of long-term stability and simple sample preparation by deposition on a regular sample plate, the use of DHB-functionalized nanoparticles combined with high-resolution MALDI-TOF MS provides a generic platform for rapid and unambiguous structure determination of small molecules. (J Am Soc Mass Spectrom 2010, 21, 1930-1939) (c) 2010 Published by Elsevier Inc. on behalf of American Society for Mass Spectrometry
\end{abstract}

$\mathrm{N}$ ew types of organic and inorganic molecules having diverse structures with unique chemical, electronic, and optoelectronic properties have demonstrated potential applications within many fields, such as medicine, material science, biosensors, energy devices, environmental engineering, and food additive processing [1-3]. Effective structural characterization of these small molecules is critical in the pipeline of design, synthetic strategy, and further development of highly efficient, commercially expedient, and highquality materials $[4,5]$. Thus, the development of an efficient high-performance screening platform for vari-

Address reprint requests to Dr. Y.-J. Chen, Institute of Chemistry, Academia Sinica, 128 Academia Road, Section 2, Nankang, Taipei 11529, Taiwan. E-mail: yjchen@chem.sinica.edu.tw ous materials is important for accurate identification of small molecular weight compounds.

Matrix-assisted laser desorption ionization time-offlight mass spectrometry (MALDI-TOF MS) has allowed for a new era in the analysis of diverse molecules because of its high sensitivity, rapid analysis, simplicity of operation, and utility for automatic high-throughput screening [6, 7]. For small molecule analysis, in principle, the use of MALDI-TOF MS should provide many benefits, including high detection sensitivity, tolerance of impurities, and simple spectra for complex samples $[1,2,7]$. Nevertheless, many shortcomings still arise due to the use of matrix material, causing abundant matrixderived interferences in the small molecular weight region $(<500 \mathrm{Da})$ of the mass spectra, suppression of analyte signals, and/or isobaric overlay with analyte peaks in the MALDI spectra [8]. To implement MALDI- 
TOF MS as a powerful tool for small molecule analysis, these drawbacks need to be eliminated.

To improve the signal of small molecules, various matrices have been developed, such as organic [9-11], inorganic [12], liquid [13-15], and surface-assisted desorption/ionization (SALDI) matrices [16-18], as well as matrix-free methods such as desorption/ionization on silicon (DIOS) [19, 20], silicon nanowires (SiNW) [21], and nanostructure-initiator mass spectrometry (NIMS) [22]. Many nanomaterials, such as carbon nanotubes [23-25], gold nanoparticles (AuNPs) [26], silicon nanoparticles [27], titanium dioxide nanoparticles $\left(\mathrm{TiO}_{2} \mathrm{NPs}\right)$ [28], and silver nanoparticles (AgNPs) [29], have been employed as ionization elements for small molecules. Due to their large surface area-to-volume ratio, these nano-sized materials provide efficient absorption and transfer of energy to the analyte and function as laser absorption matrices to provide efficient ionization in the MALDI-TOF MS analysis of small molecules. In a previous report, we demonstrated the use of MALDI matrix-functionalized magnetic $\mathrm{Fe}_{3} \mathrm{O}_{4}$ nanoparticles (abbreviated as matrix@MNP) as a background-free matrix for drug analysis with enhanced detection performance [30]. However, the ability of this new material to provide soft ionization for the identification of small molecules with diverse structures needs to be further investigated.

The main focus of this work was to develop a generic nanoparticle-assisted MALDI method for backgroundfree and high-performance MS measurements to complement or to ideally outperform other MS methods for small molecule analysis. Thus, we explored the broad applicability of matrix@MNP as an ionization matrix for rapid screening and identification of various categories of small molecules with prominent, background-free, and readily identifiable protonated ions within better than $5 \mathrm{ppm}$ mass accuracy. We also investigated the ability of DHB@MNP to enhance the precursor-ion signal, facilitating a more comprehensive product-ion fragmentation pattern for the differentiation of isomeric compounds. Finally, we also demonstrated the applicability of DHB@MNP for the rapid screening of a library of glycolipids that have low protonation ability in MALDI-TOF MS.

\section{Experimental}

\section{Materials}

Tetraethyl orthosilicate (TEOS, Sigma, St. Louis, MO, USA), 2,5-dihydrobenzoic acid (DHB, Sigma, St. Louis, MO, USA), and 25\% ammonia solution (Acros, Morris Plains, NJ, USA) were used as received. All analytes were synthesized in our Institute. Analytical grade methanol (Merck, Darmstadt, Germany), 1-propanol (Acros) and dichloromethane $\left(\mathrm{CH}^{2} \mathrm{Cl}^{2}\right)$ were used as solvents without redistillation. Deionized water (Direct-Q; Millipore SA, Molsheim, France) was also used as the solvent for both the analyte and matrix.

\section{Fabrication and Characterization of DHB@MNP}

The $\mathrm{Fe}_{3} \mathrm{O}_{4}$ MNP (30 mg) was prepared as previously reported [30] and dispersed in 1-propanol $(3 \mathrm{~mL})$ by sonication for $30 \mathrm{~min}$ at room temperature. Then, the reaction mixture was treated with $\mathrm{NH}_{4} \mathrm{OH}(25 \% \mathrm{wt} / \mathrm{wt}$, $0.35 \mathrm{~mL}), \mathrm{H}_{2} \mathrm{O}(0.3 \mathrm{~mL})$, and TEOS $(0.085 \mathrm{~mL})$, and stirred at $55^{\circ} \mathrm{C}$ for $2 \mathrm{~h}$. After filtration, the black particles $\left(\mathrm{SiO}_{2} @ \mathrm{MNP}\right)$ were washed three times with 1-propanol and three times with $\mathrm{H}_{2} \mathrm{O}$ and then added to the solution of DHB $(20 \mathrm{mg})$ in $\mathrm{H}_{2} \mathrm{O}(1 \mathrm{~mL})$ for reaction under room temperature for $12 \mathrm{~h}$. The black particles (DHB@MNP) were obtained by centrifugation (8000 $\mathrm{rpm}, 15 \mathrm{~min}$ ), washing by 1-propanol and $\mathrm{H}_{2} \mathrm{O}$ for three times, and further washing with $0.01 \mathrm{~N} \mathrm{NaOH}$ for 10 min. Finally, the black particles were washed by $\mathrm{H}_{2} \mathrm{O}$ for three times to give the DHB@MNP.

The amount of DHB on the nanoparticle surface was estimated using ultraviolet-visible spectroscopy (UVVis) spectroscopy by a Gene Quant 1300 instrument (Amersham Bioscience, Piscataway, NJ, USA). For DHB conjugation on MNP, $70 \mathrm{mg}$ of DHB (equivalent to the amount of DHB during synthesis) and $30 \mathrm{mg}$ MNP were used. Three different amounts $(90,120,270 \mathrm{mg})$ of TEOS were employed because the degree of silane polymerization on MNP can be different, thus providing different sites for DHB binding and amounts on the nanoparticle surface. For quantitation of DHB, a series of diluted DHB solutions was prepared to establish the calibration curve (supporting information Figure S-1, which can be found in the electronic version of this article). The amount of DHB conjugated on MNP was determined by subtracting the supernatant absorbance at $325 \mathrm{~nm}$, characteristic absorption wavelength for aromatic and carboxylic acid functional groups of DHB, before and after the conjugation [31]. The final densities of DHB on MNP were $914.2 \mathrm{nmol} / \mathrm{mg}$, $1314.5 \mathrm{nmol} /$ $\mathrm{mg}$, and $1981.9 \mathrm{nmol} / \mathrm{mg}$, when $90,120,270 \mathrm{mg}$ of TEOS were used, respectively. The detailed procedure is in supporting information Table S-1.

\section{Sample Preparation for MALDI-TOF MS}

DHB@MNP $(1 \mathrm{mg})$ was suspended in $0.1 \mathrm{~mL}$ of methanol to give a $10,000 \mathrm{ppm}$ stock solution, which was further diluted with methanol to give $500 \mathrm{ppm}$ for subsequent MALDI-TOF MS acquisition. In the same manner, the commercial DHB matrix $(1 \mathrm{mg})$ was dissolved in $0.1 \mathrm{~mL}$ of methanol to yield a 10,000 ppm stock solution and then diluted with methanol to 500 ppm as the working matrix solution. All organic and inorganic compounds $(1.0 \mathrm{mg})$ were dissolved in $0.5 \mathrm{~mL}$ of methanol or dichloromethane to yield a $2 \mathrm{mg} / \mathrm{mL}$ stock solution. From each analyte, $1.0 \mu \mathrm{L}$ sample solution of $5 \mu \mathrm{g} / \mathrm{mL}$ was mixed with $1.0 \mu \mathrm{L}$ DHB@MNP or commercial DHB working solution, and $1.0 \mu \mathrm{L}$ of the resulting mixture was spotted onto the stainless steel sample plate, air-dried, and analyzed in the MALDITOF MS instrument. For the laser desorption ionization 
(LDI) method, $1.0 \mu \mathrm{L}$ of the analyte $(5 \mu \mathrm{g} / \mathrm{mL})$ was directly spotted onto the stainless steel sample plate and analyzed by the MALDI-TOF MS instrument.

\section{MALDI-TOF MS Analysis}

MALDI-TOF MS spectra were obtained with either a Voyager DE-PRO MALDI-TOF MS (Applied Biosystems, Austin, TX, USA) equipped with a $\mathrm{N}_{2}$ laser (337 $\mathrm{nm})$ or an Applied Biosystems/MDS 4800 MALDITOF/TOF MS (Applied Biosystems) equipped with a Nd-YAG laser $(355 \mathrm{~nm})$ with a firing rate of $200 \mathrm{~Hz}$. The spectra obtained with the Voyager DE-PRO MALDITOF MS instrument were recorded in reflector mode using an acceleration voltage of $+20 \mathrm{kV}, 16 \mathrm{kV}$ grid voltage, $0.1 \%$ guide wire, and $100 \mathrm{~ns}$ delay time. For high-resolution MS and product-ion analysis, the spectra were recorded with an Applied Biosystems/MDS 4800 MALDI-TOF/TOF MS using an acceleration voltage of $20 \mathrm{kV}$ and grid voltage of $16 \mathrm{kV}$ in reflector mode and using CID gas (air) in the MS/MS mode. For accurate mass measurements, the instruments were calibrated with known standard (2,5-dihydrobenzoic acid, DHB) to obtain a $5 \mathrm{ppm}$ mass accuracy. The MS/MS measurements were done with a $1 \mathrm{kV}$ collision energy and a CID gas pressure of $3.7 \times 10^{-6}$ torr. Data were processed and analyzed with Data Explorer software (Applied Biosystems).

\section{Results and Discussion}

\section{Optimization and Stability of Matrix-Encapsulated Nanoparticle}

The performance of the DHB@MNP was evaluated using the organic acrylonitrile molecule, 2-[2,2]bithiophenyl-5yl-3-(4-diethylamino-phenyl)-acrylonitrile $\left(\mathrm{C}_{21} \mathrm{H}_{20} \mathrm{~N}_{2} \mathrm{~S}_{2}\right.$, $0.5 \mathrm{ng}$,) (Table 1, Compound 1) as the first model analyte. To achieve optimal ionization efficiency and minimal background interference, the ratio of TEOS/ MNP (wt/wt), which would affect the amount of DHB conjugation, was varied by changing the amount of TEOS during the silane polymerization step. With the change in TEOS/MNP ratio, a corresponding increase in the DHB amount was estimated to be $914.2 \mathrm{nmol} /$ $\mathrm{mg}, 1314.5 \mathrm{nmol} / \mathrm{mg}$ and $1981.9 \mathrm{nmol} / \mathrm{mg}$ under the three ratios of 3,6 , and $9(\mathrm{wt} / \mathrm{wt}$ ), respectively (supporting information Table S-1). Moreover, the increase or decrease in the TEOS/MNP ratio also adversely affected the size and distribution of silane polymerization on the iron oxide core nanoparticle (TEM, supporting information Figure S-2). As a consequence, high ratio $(9, \mathrm{wt} / \mathrm{wt})$ caused suppression of the $[\mathrm{M}]^{+}$by TEOS/DHB derived background peaks, while low ratio $(3, w t / w t)$ lead to incomplete ionization of Compound 1 because of lower extent of DHB conjugation (as shown in supporting information Figure S-3). It is noted that with an $8.4 \%$ RSD routinely obtained, sample homogeneity is improved by DHB@MNP, verifying that the different signal intensities obtained under different

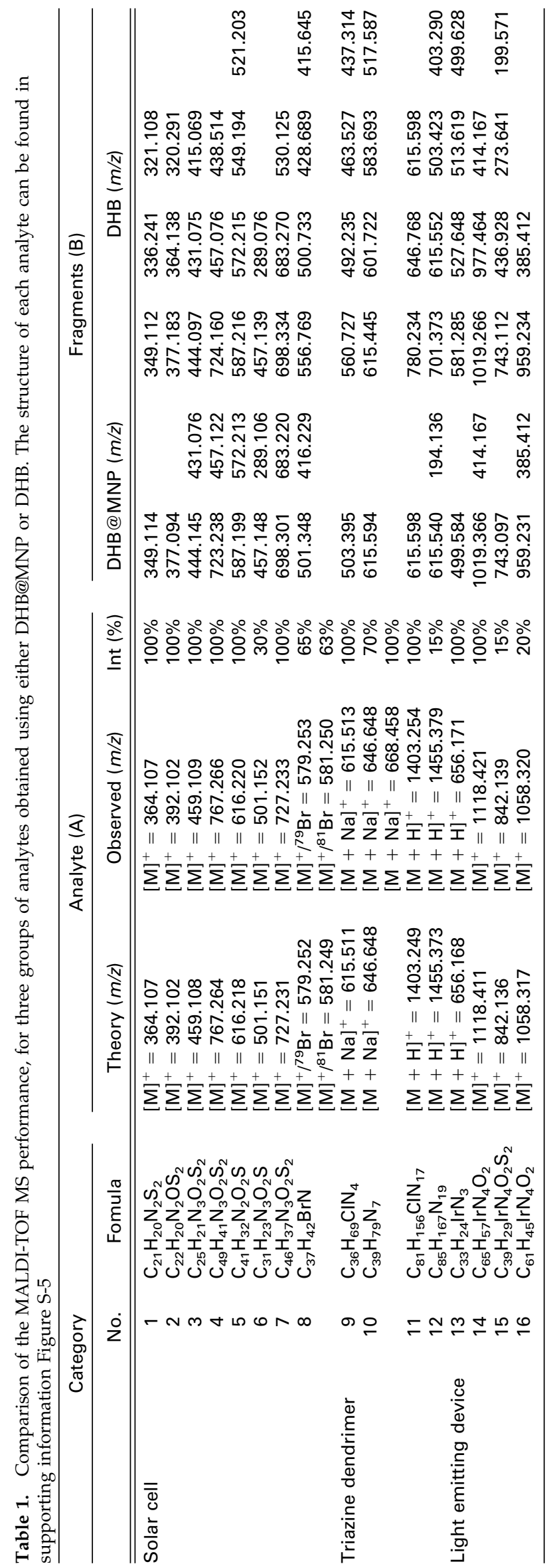


TEOS/MNP ratio is not caused by "sweet spot" effect. Hence, an optimal TEOS/MNP ratio of 6 was used for the fabrication of DHB@MNP. In addition, in routine MALDI-TOF MS analysis, preparation of fresh matrix is required to give optimal signals because synthesized matrix maybe prone to degradation after storage for a period of time. Therefore, the stability of DHB@MNP was examined after 6 and 12 mo of storage in methanol at $4{ }^{\circ} \mathrm{C}$. As shown in supporting information Figure S-4, the signal intensity of the molecular ion $[\mathrm{M}]^{+}$was conserved in all three spectra measured under identical conditions, indicating stable performance of DHB@MNP even beyond $12 \mathrm{mo}$ of storage.

\section{Soft and Enhanced Ionization by DHB@MNP}

In general, the signal intensity in MALDI depends on the choice of a good matrix that shows strong absorp- tion of laser energy and efficient proton transfer to the analyte [32]. In DHB@MNP, various substances, including the metal oxide core, $\mathrm{SiO}_{2}$ derived from silanation on the MNP, and covalently surface-bound matrix molecules, may contribute to the ionization of the analyte to different extents [30]. Although DHB@MNP contains multiple components to improve the detection of the analyte, such efficient energy absorption and transfer may also induce fragmentation of analytes with fragile structures. Thus, we investigated the intriguing interplay between ionization efficiency and the extent of fragmentation of fragile analytes by employing a direct laser desorption ionization (LDI), conventional DHB matrix, and DHB@MNP matrix in MALDI-TOF MS.

Figure 1a revealed that MALDI ionization using DHB@MNP produced only a sharp and intense molecular ion peak $[\mathrm{M}]^{+}$, a dramatic reduction of matrixderived interferences along the low molecular weight


Figure 1. The signal intensity of Compound $1(5 \mu \mathrm{g} / \mathrm{mL})$ generated by four different ionization methods. (a) MALDI ionization with nanoparticle matrix (DHB@MNP). The insert shows the mass spectrum obtained by laser desorption ionization, which was magnified 40-fold to show the molecular ion. (b) MALDI ionization with the commercial DHB matrix. (c) Fast atom bombardment ionization with 3-nitrobenzyl alcohol matrix. (d) Electrospray ionization. $[\mathrm{M}]^{+}=$analyte ion; $[\mathrm{F}]^{+}=$fragment ions; ${ }^{*}=$ matrix ions. 
range, and negligible fragmentation in contrast to the other ionization methods (Figure $1 b-d$ ). Although Compound 1 contains laser-absorbing aromatic structure, the intensity of the analyte ion produced by LDI (the insert in Figure 1a) was significantly lower than that in the presence of DHB@MNP. In conventional MALDI ionization using the commercial matrix DHB (Figure 1b), many DHB-derived peaks and strong fragment ion signals interfere with the interpretation of the spectral data. Likewise, fast atom bombardment (FAB, Figure 1c) and electrospray (ESI, Figure 1d) ionization methods resulted in abundant background ions from either the glycerol-derived matrix peak or the impurity ions present in the solvent (designated by ${ }^{*}$ ), respectively. Such extensive fragmentation sometimes leads to the disappearance of the molecular ion peak, causing difficulty in obtaining accurate mass measurements.

Taken together, with the use of DHB@MNP, we demonstrated relatively soft and enhanced ionization for Compound 1 compared with the ESI, FAB, and conventional MALDI methods. This improved ionization performance may be due to the synergistic energy absorbing effects of the magnetic nanoparticle, silane polymerization, and the organic matrix on its surface, resulting in efficient energy-transfer and relaxation for soft ionization of the fragile analyte [33, 34].

\section{General Applicability of DHB@MNP to Organic and Inorganic Molecules}

To evaluate the general applicability of the developed matrix-free ionization material, we further used DHB@MNP to analyze various types of organic and inorganic analytes with diverse structures, including inorganic light-emitting molecules, solar cell materials, and dendrimers (Table 1, see structures in supporting information Figure S-5). Each group consisted of several compounds with the same core structure. These molecules have been widely applied in material and biomedical sciences, and they have inspired the development of synthetic strategies.

For the analysis of 16 compounds, as expected, the matrix-derived background intensity obtained by the use of commercial DHB matrix results in $>2 \sim 60$-fold higher than that of DHB@MNP (Figure 2a). More sig-

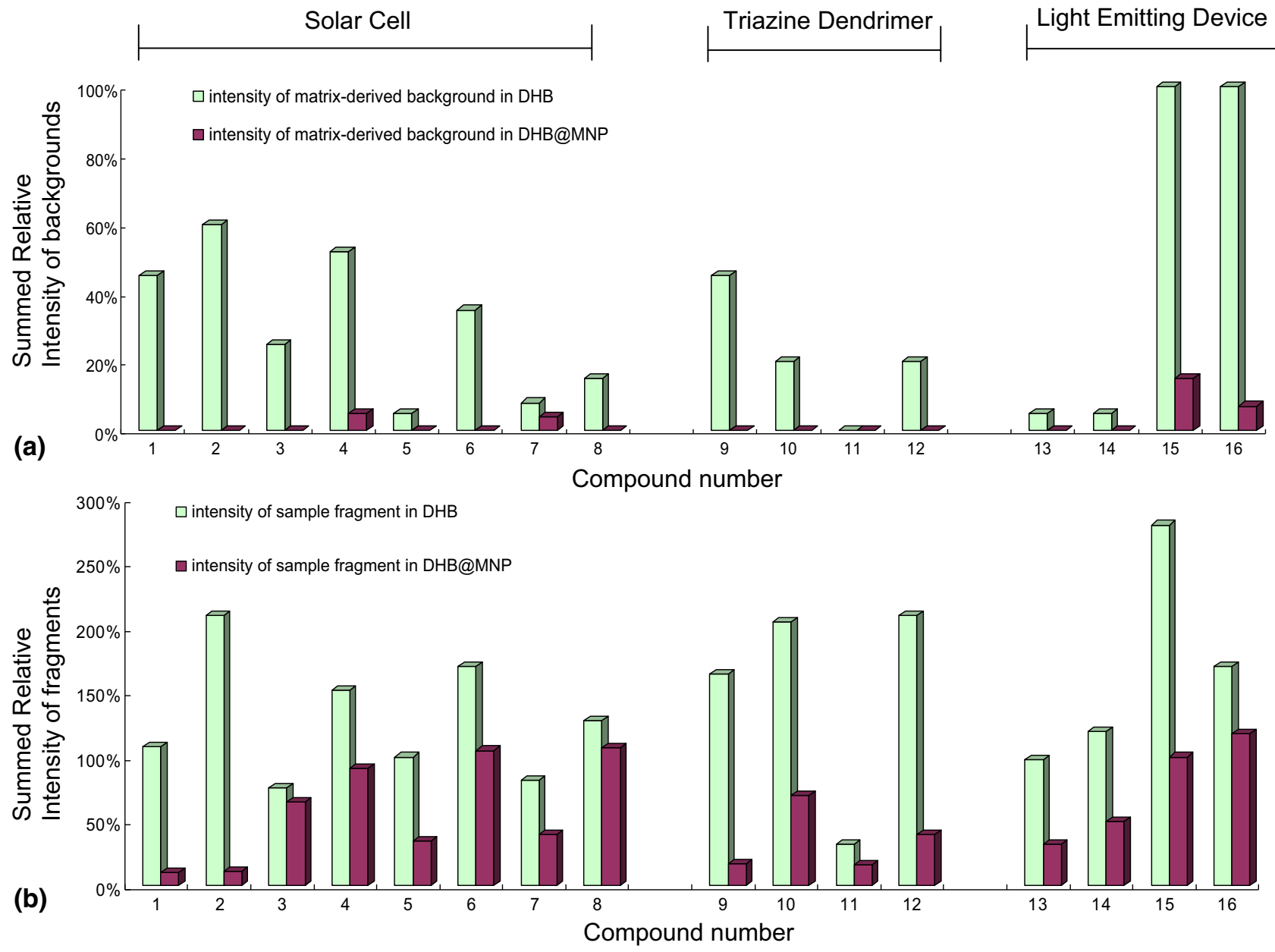

Figure 2. Comparison of background and fragments using DHB and DHB@MNP in MALDI MS. The sum of relative intensities of (a) DHB or DHB@MNP derived backgrounds and (b) fragments of the compounds tested in Table 1. The compound number corresponds to those in Table 1. 
nificantly, the DHB@MNP generated much softer ionization; the total intensities of fragments from each analyte reduced by $2 \sim 20$-fold compared with the analyte fragment intensities obtained by using commercial DHB (Figure 2b). In Table 1, the first group (Compounds 1-8), consists of a series of dipolar dyes and their precursors, containing a cyanovinyl (1-4) or a pyrrole-based conjugate (5-8) linker, is used as dyesensitized solar cells (DSSCs) [35]. DHB@MNP allows strong signals for most of the molecular ions in Compounds $1,2,3,4,5$, and 7 (100\% relative intensities) (Table 1). In the comparison to matrix-derived background signals, DHB@MNP produced fewer than five matrix peaks ( $\leq 5 \%$ intensity), whereas the use of commercial matrix resulted in 4-30 intense background peaks for almost all of the compounds $(5 \%-60 \%$ relative intensities, the detail was in supporting information Table S-2). Furthermore, compared with conventional matrix DHB, the use of DHB@MNP produced signifi- cantly fewer fragment ions as shown in Figure $2 b$ for Compounds 1, 2, 5, and 6 .

In the characterization of synthetic small molecules, a molecular mass measured within accuracy better than $5 \mathrm{ppm}$ is often sufficient to confirm the molecular formula of a compound [36]. The results listed in Table 1 show that all of the molecular ions have $5 \mathrm{ppm}$ accuracy comparable to the theoretical mass. Furthermore, in Compound $8\left(\mathrm{C}_{37} \mathrm{H}_{42} \mathrm{BrN}\right)$, the isotopic distribution of the bromine atom, ${ }^{79} \mathrm{Br}$ and ${ }^{81} \mathrm{Br}$, was explicitly observed in the molecular ion peaks, $\left[\mathrm{M}^{79} \mathrm{Br}\right]^{+}$and $\left[\mathrm{M}^{81} \mathrm{Br}\right]^{+}$, and it closely resembles the predicted isotope pattern (supporting information Figure S-6).

The second group (Compounds 9-12 in Table 1), consisting of triazine-based dendritic nanomaterials, is widely used in drug delivery and catalytic materials, liquid crystals, and biosensors [37-39]. The third group (Compounds 13-16 in Table 1), consisting of phosphorescent cyclometalated iridium complexes, has received


$1, m / z 349$

Figure 3. Comparison of MS/MS spectrum of Compound $1\left([\mathrm{M}]^{+}=364.107,5 \mu \mathrm{g} / \mathrm{mL}\right)$ obtained using the DHB@MNP matrix and the commercial DHB matrix (insert). The assignments of the major fragment peaks in the spectra correspond to the numbering of the fragmentation pattern shown in the structure of Compound 1. 
extensive attention for potential use in the fabrication of organic light-emitting devices (OLEDs) due to its easy preparation from iridium precursors and its high phosphorescence efficiency $[40,41]$. We expected that using the soft ionization of $\mathrm{DHB} @ \mathrm{MNP}$ to obtain accurate masses of their intact structures would allow differentiation of these synthetic variants. As shown in Table 1, the observation of predominant molecular ions within an accuracy of 5 ppm demonstrated the success and general applicability of DHB@MNP for accurate mass measurements of the two groups of materials with negligible background or matrix-derived noise. Notably, the numbers of fragments were also reduced by using DHB@MNP as the matrix, consistent with the previous observation for group 1 (Figure $2 b$ ). In conclusion, DHB@MNP-assisted MALDI-TOF MS provided direct, soft, and accurate mass measurements for novel organic and organometallic compounds.

\section{Structural Characterization by Enhanced Product-Ion Analysis}

Novel matrix-free materials have been developed for analysis of small molecules by MALDI, their performances on product-ion analysis have lower sensitivity than the use of conventional organic matrices $[1,2]$. Therefore, we examined and compared the MS/MS performance of DHB@MNP with DHB matrix for structural characterization of the thermally labile Compound 1. As shown in Figure 3, significantly comprehensive product-ion spectra and stronger fragment peak signals were generated with DHB@MNP (Figure 3), whereas the commercial DHB matrix only produced few weak fragment ions (the insert of Figure 3). DHB@MNP yielded about 2-fold more intense molecular ion signal (Figure 1a) than that produced using the commercial matrix DHB (Figure 1b). However, such difference did not fully account for the dramatically different extent of fragmentation in Figure 3. We believe that these superior results may be due to both good ionization and efficient energy absorption of the precursor ion in DHB@MNP-assisted MALDI-TOF MS. As shown in Figure 3, the fragmentation corresponds to the loss of the first $\mathrm{CH}_{3}$ group $\left[\mathrm{C}_{20} \mathrm{H}_{17} \mathrm{~N}_{2} \mathrm{~S}_{2}\right]^{+}$(349 Da), and then the ethyl group $\left[\mathrm{C}_{19} \mathrm{H}_{15} \mathrm{~N}_{2} \mathrm{~S}_{2}\right]^{+}$(336 Da). Subsequent cleavage of the diethyl group on the amine allowed the formation of the fragment ion $\left[\mathrm{C}_{17} \mathrm{H}_{11} \mathrm{~N}_{2} \mathrm{~S}_{2}\right]_{+}$(mass 307 Da). After cleavage of the dithiophene and diethylamine groups, the final product, 3-phenyl-acrylonitrile (128 Da), was observed. These results demonstrated that DHB@MNP provided a better product-ion performance than the use of commercial DHB.

Encouraged by the success of DHB@MNP in the product-ion experiments, we then evaluated the performance of DHB@MNP to differentiate the isomeric compounds, prometryn and terbutryn as model analytes. Monitoring of residual traces of these pesticides is indispensable to evaluate their impact on human health and the environment $[42,43]$. For rapid identification of two isomeric triazines, MALDI-TOF/TOF MS analysis with DHB@MNP was performed on the molecular ions of terbutryn and prometryn at $m / z 242$ (insert in Figure 4).
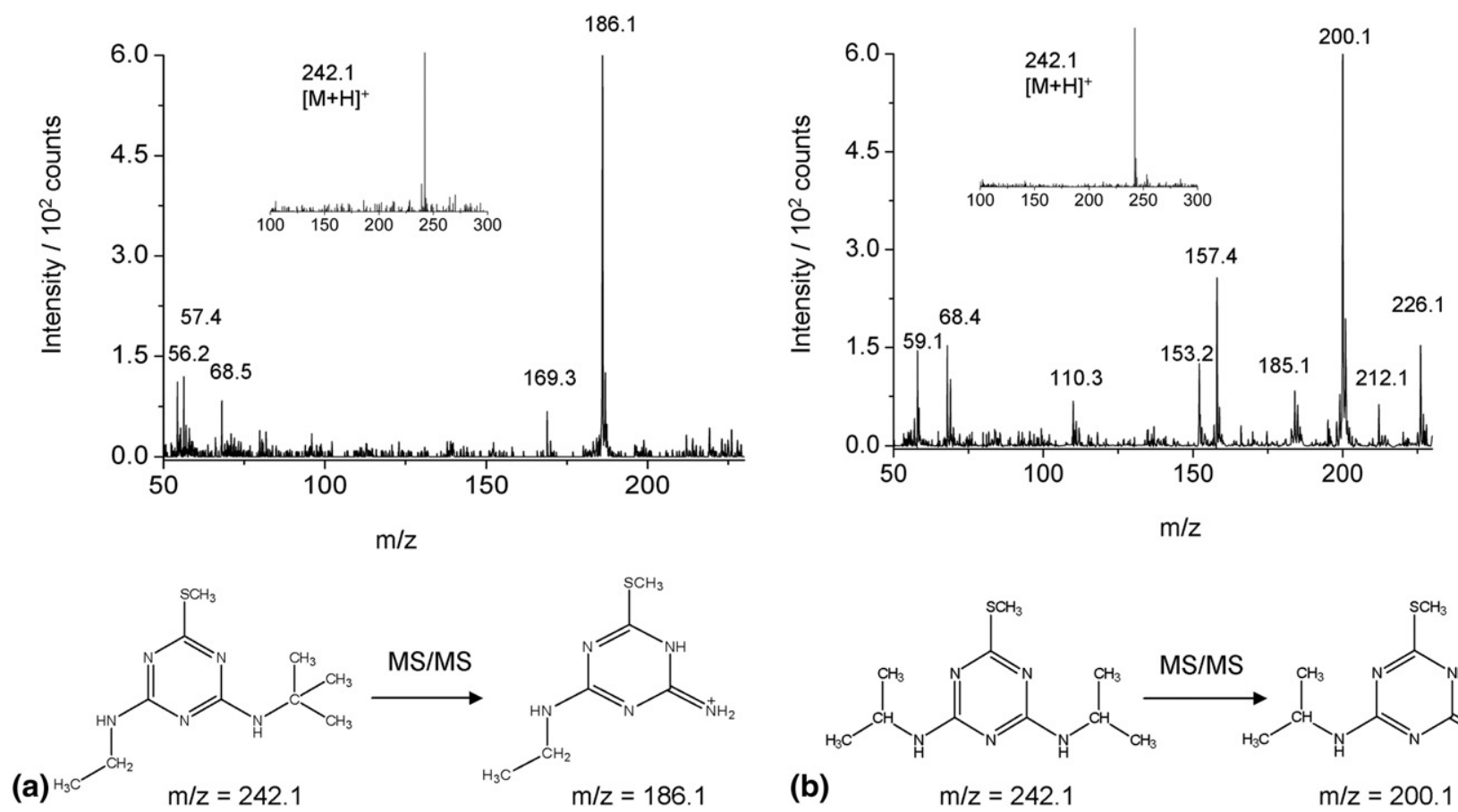<smiles>CC(C)Nc1nc(NC(C)C)nc(NC(C)C)n1</smiles>

Figure 4. MALDI TOF/TOF spectrum of two triazine isomers $(5 \mu \mathrm{g} / \mathrm{mL})$, (a) terbutryn and (b) prometryn, obtained using DHB@MNP matrix. The inserts show the precursor ion $=[\mathrm{M}+\mathrm{H}]^{+}$. The dissociation of the precursor ion to the major product ion is shown below each spectrum. 
Figure $4 \mathrm{a}$ and $\mathrm{b}$ show the product-ion spectra and fragmentation assignments for terbutryn and prometryn, respectively. A comparison of the product-ion spectra of both compounds revealed that terbutryn showed unique fragment ions at $\mathrm{m} / \mathrm{z} 186$ and 169 (Figure 4a), corresponding to the loss of tert-butyl and tertbutylamine groups, respectively (see fragment structures in supporting information schemes S-1 and S-2). In contrast, the product-ion spectrum of prometryn contained a unique fragment at $\mathrm{m} / \mathrm{z} 200$, corresponding to the loss of one isopropyl radical, and leading to the formation of the dealkylated derivatives. In addition, other minor fragments at $m / z 226,212,185,157,153,110$, and 59 were uniquely observed in the product-ion spectrum of prometryn (Figure 4b). Although prometryn and terbutryn have the same precursor ion, they were identified unambiguously by their unique product ions.

The post-source-decay (PSD)-MALDI had been used to characterize these two triazine isomers, and the results showed similar fragmentation patterns for both isomers, but only two characteristic ions at $\mathrm{m} / \mathrm{z} 186$ and 200 were observed to differentiate their structures [44]. Thus, we conclude that high-energy MALDI-CID com- bined with DHB@MNP facilitates refined fingerprint identification of isomeric compounds by enhanced product-ion fragmentation.

\section{Rapid Screening of Glycolipid Library}

We applied DHB@MNP to the rapid analysis of a small library of twelve $\alpha$-galactosyl lipids [45, 46]. The structural difference among glycolipids is only in the lipid moiety, which contains various saturated carbon lengths (structures shown in supporting information Figure S-7). Thus, it was impractical to use ${ }^{1} \mathrm{H}$ NMR for structure analysis, whereas mass analysis could provide useful information on lipid length based on differences in molecular weight. However, carbohydrates have low protonation efficiency [25] and alkali metal salts are often employed to facilitate ionization [47]. In matrix@MNP-assisted MALDI-TOF MS analysis, despite the low protonation tendency, as shown in Figure 5 , all of these $\alpha$-galactosyl lipids exhibited clean mass spectra displaying strong sodium-adducted molecular ion peaks $[\mathrm{M}+\mathrm{Na}]^{+}$, a characteristic set of mass spectrometric signals exhibited by carbohydrates in MALDI-TOF MS. Detection of alkali metal-adducted



Figure 5. A group of 12 structurally similar glycolipids $(5 \mu \mathrm{g} / \mathrm{mL})$ were screened by MALDI-TOF MS with the DHB@MNP matrix. $[\mathrm{M}+\mathrm{Na}]^{+}$and $[\mathrm{F}]^{+}$correspond to the sodium-adducted peaks and fragment ions, respectively. 
peaks is common in the use of matrix-free materials, such as Au nanoparticles [48], DIOS [49], carbon nanotubes [25], and matrix enhanced laser desorption/ionization (MELDI) materials $[50,51]$. In our approach, the ability of DHB@MNP to efficiently enhance the signal of glycolipids may be due to the incorporated residual sodium ion from the washing step with $\mathrm{NaOH}$ during DHB@MNP synthesis. Notably, all the spectra showed excellent signal-to-noise ratios $(>30)$, and only a few galactose-derived fragment ions were observed in Compounds $1,6,9$, and 11 . Due to the rapid acquisition of MALDI-TOF MS, the analysis of these 12 glycolipids was completed within $10 \mathrm{~min}$, including the matrix preparation, analyte/DHB@MNP deposition on MALDI plate, and data acquisition.

\section{Conclusions}

In this study, we demonstrated the general applicability of DHB@MNP as a background-free and soft ionization matrix in the analysis of structurally diverse organic and inorganic compounds with low molecular weight. By the synergetic effects of energy-absorbing MNP and surface DHB, the use of DHB@MNP allows soft and enhanced ionization to obtain accurate mass measurements of fragile compounds. The effect of the size, TEOS/MNP uniformity, and distribution on the ionization efficiency of DHB@MNP and their mechanism of energy-transfer will be further investigated. In addition, the unique features of $\mathrm{DHB} @ \mathrm{MNP}$ also lead to the enhancement of fragment signals and superior fragmentation patterns in MS/MS mode for unambiguous small molecule identification or structural elucidation of isomeric compounds. These advantages, combined with the automatic MALDI-TOF MS acquisition, make DHB@MNP an attractive matrix for high-throughput screening of novel materials. The sample preparation is simple and similar to the standard MALDI procedure of deposition on a regular MALDI sample plate. With the advantages of long-term stability, simple and rapid analysis, and automated high-throughput screening, the matrix@MNP-assisted MALDI-TOF MS provides an easy-to-use alternative that is potentially useful for drug discovery, development, and quality control monitoring in drug manufacturing. To further extend the application of DHB@MNP, the fabrication of multifunctional nanoparticles having both the matrix and extraction functionalities for small molecules is ongoing. Homogeneous sample preparation with the nanoparticle will require further studies for fast and accurate quantification of small molecules.

\section{Acknowledgments}

The authors acknowledge support for this work by the Institute of Chemistry in Academia Sinica, National Tsing Hua University, and Academia Sinica Research Project on Nanoscience and Technology. The authors also thank Miss Chiu-Yun Chen for helping prepare the manuscript.

\section{Appendix A Supplementary Material}

Supplementary material associated with this article may be found in the online version at doi:10.1016/j.jasms. 2010.08.001.

\section{References}

1. Cohen, L.; Gusev, A. Small Molecule Analysis by MALDI Mass Spectrometry. Anal. Bioanal. Chem. 2002, 373, 571-586.

2. Hillenkamp, F.; Peter-Katalinic, J. MALDI MS, a Practical Guide to Instrumentation, Methods, and Applications; WILEY-VCH Verlag $\mathrm{GmbH}$ and Co. KGaA: Weinheim, 2007; p. 299.

3. Wagner, B. K.; Carrinski, H. A.; Ahn, Y. H.; Kim, Y. K.; Gilbert, T. J.; Fomina, D. A.; Schreiber, S. L.; Chang, Y. T.; Clemons, P. A. SmallMolecule Fluorophores to Detect Cell-State Switching in the Context of High-Throughput Screening. J. Am. Chem. Soc. 2008, 130, 4208-4209.

4. Becker, J. S.; Dietze, H. J. State-of-the-Art in Inorganic Mass Spectrometry for Analysis of High-Purity Materials. Int. J. Mass Spectrom. 2003, $228,127-150$.

5. Ham, R. V.; Vaeck, L. V.; Adriaens, A.; Adams, F. Static Secondary ion Mass Spectrometry for Organic and Inorganic Molecular Analysis in Solids. Anal. Chem. Acta 2003, 500, 259-278.

6. Sleno, L.; Volmer, D. A. Some Fundamental and Technical Aspects of the Quantitative Analysis of Pharmaceutical Drugs by Matrix-Assisted Laser Desorption/Ionization Mass Spectrometry. Rapid Commun. Mass Spectrom. 2005, 19, 1928-1936.

7. Zhang, H.; Cha, S.; Yeung, E. S. Colloidal Graphite-Assisted Laser Desorption/Ionization MS and $\mathrm{MS}^{n}$ of Small Molecules. 2. Direct Profiling and MS Imaging of Small Metabolites from Fruits. Anal. Chem. 2007, 79, 6575-6584.

8. Guo, Z.; Zhang, Q.; Zou, H.; Guo, B.; Ni, J. A. Method for the Analysis of Low-Mass Molecules by MALDI-TOF Mass Spectrometry. Anal. Chem. 2002, 74, 1637-1641.

9. Jones, R. M.; Lamb, J. H.; C. K. Lim. Urinary Porphyrin Profiles by Laser Desorption/Ionization Time-of-Flight Mass Spectrometry Without the Use of Classical Matrices. Rapid Commun. Mass Spectrom. 1995, 9, 921-923.

10. Ayorinde, F. O.; Hambright, P.; Porter, T. N.; Keith, Q. L. Use of MesoTetrakis(Pentafluorophenyl)Porphyrin as a Matrix for low Molecular Weight Alkylphenol Ethoxylates in Laser Desorption/Ionization Timeof-Flight Mass Spectrometry. Rapid Commun. Mass Spectrom. 1999, 13, 2474-2479.

11. Kang, M. J.; Tholey, A.; Heinzle, E. Quantitation of Low Molecular Mass Substrates and Products of Enzyme Catalyzed Reactions Using MatrixAssisted Laser Desorption/Ionization Time-of-Flight Mass Spectrometry. Rapid Commun. Mass Spectrom. 2000, 14, 1972-1978.

12. Kinumi, T.; Saisu, T.; Takayama, M.; Niwa, H. Matrix-Assisted Laser Desorption/Ionization Time-of-Flight Mass Spectrometry Using an Inorganic Particle Matrix for Small Molecule Analysis. J. Mass Spectrom. 2000, 35, 417-422.

13. Dominic Chan, T. W.; Thomas, I.; Colburn, A. W.; Derrick, P. J. Initial Velocities of Positive and Negative Protein Molecule-Ions Produced in Matrix-Assisted Ultraviolet Laser Desorption Using a Liquid Matrix. Chem. Phys. Lett. 1994, 222, 579-585.

14. Williams, J. B.; Gusev, A. I.; Hercules, D. M. Use of Liquid Matrices for Matrix-Assisted Laser Desorption Ionization of Polyglycols and Poly(Dimethylsiloxanes). Macromolecules 1996, 29, 8144-8150.

15. Mank, M.; Stahl, B.; Boehm, G. 2,5-Dihydroxybenzoic Acid Butylamine and Other Ionic Liquid Matrixes for Enhanced MALDI-MS Analysis of Biomolecules. Anal. Chem. 2004, 76, 2938-2950.

16. Chen, Y. C.; Tsai, M. F. Sensitivity Enhancement for Nitrophenols Using Cationic Surfactant-Modified Activated Carbon for Solid-Phase Extraction Surface-Assisted Laser Desorption/Ionization Mass Spectrometry. Rapid Commun. Mass Spectrom. 2000, 14, 2300-2304.

17. Wu, J. Y.; Chen, Y. C. A Novel Approach of Combining Thin-Layer Chromatography with Surface-Assisted Laser Desorption/Ionization (SALDI) Time-of-Flight Mass Spectrometry. J. Mass Spectrom. 2002, 37, 85-90.

18. Finkel, N. H.; Prevo, B. G.; Velev, O. D.; He, L. Ordered Silicon Nanocavity Arrays in Surface-Assisted Desorption/Ionization Mass Spectrometry. Anal. Chem. 2005, 77, 1088-1095.

19. Wei, J.; Buriak, J. M.; Siuzdak, G. Desorption/Ionization Mass Spectrometry on Porous Silicon. Nature 1999, 399, 243-246.

20. Trauger, S. A.; Go, E. P.; Shen, Z. J.; Apon, V.; Compton, B. J.; Bouvier, E. S.; Finn, M. G.; Siuzdak, G. High Sensitivity and Analyte Capture with Desorption/Ionization Mass Spectrometry on Silylated Porous Silicon. Anal. Chem. 2004, 76, 4484-4489.

21. Go, E. P.; Apon, J. V.; Luo, G.; Saghatelian, A.; Daniels, R. H.; Sahi, V.; Dubrow, R.; Cravatt, B. F.; Vertes, A.; Siuzdak, G. Desorption/Ionization on Silicon Nanowires. Anal. Chem. 2005, 77, 1641-1646.

22. Northen, T. R.; Yanes, O.; Northen, M. T.; Marrinucci, D.; Uritboonthai, W.; Apon, J.; Golledge, S. L.; Nordstrom, A.; Siuzdak, G. Clathrate Nanostructures for Mass Spectrometry. Nature 2007, 449, 1033-1036. 
23. Xu, S.; Li, Y.; Zou, H.; Qiu, J.; Guo Z.; Guo, B. Carbon Nanotubes as Assisted Matrix for Laser Desorption/Ionization Time-of-Flight Mass Spectrometry. Anal. Chem. 2003, 75, 6191-6195.

24. Pan, C.; Xu, S.; Hu, L.; Su, X.; Ou, J.; Zou, H.; Guo, Z.; Zhang Y.; Guo, B. Using Oxidized Carbon Nanotubes as Matrix for Analysis of Small Molecules by MALDI-TOF MS. J. Am. Soc. Mass Spectrom. 2005, 16, 883-892.

25. Ren, S. F.; Zhang, L.; Cheng, Z. H.; Guo, Y. L. Immobilized Carbon Nanotubes as Matrix for MALDI-TOF MS Analysis: Applications to Neutral Small Carbohydrates. J. Am. Soc. Mass Spectrom. 2005, 16, 333-339.

26. Huang, Y. F.; Chang, H. T. Nile Red-Adsorbed Gold Nanoparticle Matrixes for Determining Aminothiols Through Surface-Assisted Laser Desorption/Ionization Mass Spectrometry. Anal. Chem. 2006, 78, 14851493.

27. Wen, X.; Dagan, S.; Wysocki, V. H. Small-Molecule Analysis with Silicon-Nanoparticle-Assisted Laser Desorption/Ionization Mass Spectrometry. Anal. Chem. 2007, 79, 434-444.

28. Yuan, M.; Shan, Z.; Tian, B.; Tu, B.; Yang, P.; Zhao, D. Preparation of Highly Ordered Mesoporous $\mathrm{WO}^{3}-\mathrm{TiO}^{2}$ as Matrix in Matrix-Assisted Laser Desorption/Ionization Mass Spectrometry. Micropor. Mesopor. Mater. 2005, 78, 37-41.

29. Kamlesh, S.; Hui-Fen, W. Applications of Silver Nanoparticles Capped with Different Functional Groups as the Matrix and Affinity Probes in Surface-Assisted Laser Desorption/Ionization Time-of-Flight and Atmospheric Pressure Matrix-Assisted Laser Desorption/Ionization ion Trap Mass Spectrometry for Rapid Analysis of Sulfur Drugs and Biothiols in Human Urine. Rapid Commun. Mass Spectrom. 2008, 22, 2863-2872.

30. Lin, P. C.; Tseng, M. C.; Su, A. K.; Chen, Y. J.; Lin, C. C. Functionalized Magnetic Nanoparticles for Small-Molecule Isolation, Identification, and Quantification. Anal. Chem. 2007, 79, 3401-3408.

31. Allwood, D. A.; Dreyfus, R. W.; Perera, I. K.; Dyer, P. E. UV Optical Absorption of Matrices Used for Matrix-Assisted Laser Desorption/ Ionization. Rapid Commun. Mass Spectrom. 1996, 10, 1575-1578.

32. Knochenmuss, R. Ion Formation Mechanisms in UV-MALDI. Analyst 2006, 131, 966-986.

33. Chen, L. X.; Liu, T. Thurnauer, M. C. Csencsits, R. Rajh, T. $\mathrm{Fe}^{2} \mathrm{O}^{3}$ Nanoparticle Structures Investigated by X-ray Absorption Near-Edge Structure, Surface Modifications, and Model Calculations. J. Phys. Chem. B. 2002, 106, 8539-8546.

34. Donatti, D. A.; Ruiz, A. I.; De Moraes, F. G.; Vollet, D. R. UV-Visible Absorption Characteristics of TEOS-Derived and Cr-Doped Silica Sonogels Aged in Different $\mathrm{pH}$ Solution and Heat Treated up to $600{ }^{\circ} \mathrm{C}$. J. J. Sol-Gel. Sci. Technol. 2003, 28, 31-35.

35. Yen, Y. S.; Hsu, Y. C.; Lin, J. T.; Chang, C. W.; Hsu C. P.; Yin, D. J. Pyrrole-Based Organic Dyes for Dye-Sensitized Solar Cells. J. Phys. Chem. C 2008, 112, 12557-12567.

36. Balogh, M. P. Debating Resolution and Mass Accuracy in Mass Spectrometry. Spectroscopy 2004, 19, 34-40.
37. Tsiourvas, D.; Felekis, T.; Sideratou, Z.; Paleos, C. M. Liquid Crystals Derived from Cholesterol Functionalized Poly(Propylene Imine) Dendrimers. Macromolecules 2002, 35, 6466-6469.

38. Minard-Basquin, C.; Weil, T.; Hohner, A.; Radler J. O.; Mullen, K. A Polyphenylene Dendrimer-Detergent Complex as a Highly Fluorescent Probe for Bioassays. J. Am. Chem. Soc. 2003, 125, 5832-5838.

39. Lai, L. L.; Wang, L. Y.; Lee, C. H.; Lin Y. C.; Cheng, K. L. Nanomaterials of Triazine-Based Dendrons:Convergent Synthesis and Their Physical Studies. Org. Lett. 2006, 8, 1541-1544.

40. Huang, W. S.; Lin, J. T.; Chien, C. H.; Tao, Y. T.; Sun, S. S.; Wen, Y. S. Highly Phosphorescent Bis-Cyclometalated Iridium Complexes Containing Benzoimidazole-Based Ligands. Chem. Mater. 2004, 16, 2480 2488

41. Scholz, S.; Corten, C.; Walzer, K.; Kuckling, D.; Leo, K. Photochemica Reactions in Organic Semiconductor Thin Films. Org. Electron. 2007, 8 , 709-717.

42. Evgenidou, E.; Fytianos, K. Photodegradation of Triazine Herbicides in Aqueous Solutions and Natural Waters. J. Agric. Food Chem. 2002, 50 6423-6427.

43. Ibanez, M.; Sancho, J. V.; Pozo, O. J.; Hernandez, F. Use of Quadrupole Time-of-Flight Mass Spectrometry in Environmental Analysis: Elucidation of Transformation Products of Triazine Herbicides in Water after UV Exposure. Anal. Chem. 2004, 76, 1328-1335.

44. Cheng, Y.; Hercules, D. M. Quantitative Estimation of Geometric Isomers by Post-Source Decay MALDI MS. Microchem. J. 2002, 72 255-267.

45. Hung, L. C.; Lin, C. C.; Hung, S. K.; Wu, B. C.; Jan, M. D.; Liou, S. H. $\mathrm{Fu}, \mathrm{S}$. L. A Synthetic Analog of $\alpha$-Galactosylceramide Induces Macrophage Activation Via the TLR4-signaling Pathways. Biochem. Pharmacol. 2007, 73, 1957-1970.

46. Fan, G. T.; Pan, Y. S.; Lu, K. C.; Cheng, Y. P.; Lin, W. C.; Lin, S.; Lin C. H.; Wong, C. H.; Fang, J. M.; Lin, C. C. Synthesis of $\alpha$-Galactosyl Ceramide and the Related Glycolipids for Evaluation of Their Activities on Mouse Splenocytes. Tetrahedron 2005, 61, 1855-1862.

47. Harvey, D. J. Matrix-Assisted Laser. Desorption/Ionization Mass Spectrometry of Carbohydrates. Mass Spectrom. Rev. 1999, 18, 349-450.

48. Su, C. L.; Tseng, W. L. Gold Nanoparticles as Assisted Matrix for Determining Neutral Small Carbohydrates through Laser Desorption/ Ionization Time-of-Flight Mass Spectrometry. Anal. Chem. 2007, 79 1626-1633.

49. Li, Q.; Ricardo, A.; Benner, S. A.; Winefordner, J. D.; Powell, D. H. Desorption/Ionization on Porous Silicon Mass Spectrometry Studies on Pentose-Borate Complexes. Anal. Chem. 2005, 77, 4503-4508.

50. Muhammad Ahsan, H.; Guenther, S.; Rania, B.; Saowapak, K.; Bernhard, B.; Isabel, F.; Gudrun, A.; Michael, P.; Ortwin, B.; Guenther, K. B. Identification of Carbohydrates by Matrix-Free Material-Enhanced Laser Desorption/Ionization Mass Spectrometry. Rapid Commun. Mass Spectrom. 2007, 21, 2759-2769.

51. Hashir, M. A.; Stecher, G.; Mayr S.; Bonn, G. K. Identification of Amino Acids by Material Enhanced Laser Desorption/Ionization Mass Spectrometry (MELDI-MS) in Positive- and Negative-Ion Mode. Int. J. Mass Spectrom. 2009, 279, 15-24. 